\title{
Breast cancer in young women
}

\section{Part III: Systemic treatment}

\author{
Agustí Barnadas · Carlos Vázquez
}

Received: 1 July 2010/ Accepted: 6 July 2010/Published online: 14 August 2010

(C) Springer Science+Business Media, LLC. 2010

\begin{abstract}
Systemic treatment in young women with early or advanced breast cancer is a challenging issue. Since it is not clear whether women under 30-40 years should be treated differently from older women based on age alone, current treatment is based on chemotherapy, endocrine therapy, targeted therapy, or a combination of the three. In young women the wish to preserve the breast and maintain reproductive function are crucial aspects to evaluate before deciding on the type of therapy. Neoadjuvant therapy, which should include taxanes and/or anthracyclines, is indicated in young women with operable tumors in whom, on initial consideration, breast conserving surgery cannot be performed. Adjuvant chemotherapy is considered in women with triple-negative disease who are at sufficient risk of relapse and in patients with HER-2 positive tumors, together or before administration of trastuzumab, and
\end{abstract}

adjuvant endocrine therapy is indicated in endocrinereceptor positive disease. Abolition/suppression of ovarian function is a primary objective in both early and advanced breast cancer, the best option for this purpose being luteinizing hormone-releasing hormone agonists combined with tamoxifen. As in early disease, treatment of young women with advanced breast cancer does not differ from that of their older counterparts, and mainly includes the use of anthracyclines, taxanes, vinorelbine, gemcitabine, paclitaxel, and capecitabine. Targeted therapies such as trastuzumab, lapatinib, and bevacizumab should be used in combination with chemotherapy according to the latest results reported in randomized trials.

Conflict of Interest The authors have no conflict of interest.

\footnotetext{
A. Barnadas $(\square)$

Hospital Santa Creu i Sant Pau, Barcelona, Spain

e-mail: abarnadasm@santpau.cat

C. Vázquez

Instituto Valenciano de Oncología, Valencia, Spain

e-mail: cvazquez@fivo.org
} 